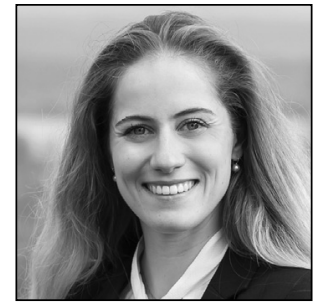

Julia Florian*1

Wissenschaftliche Mitarbeiterin Lehrstuhl für Bürgerliches Recht, Zivilprozessrecht, Internationales Privatrecht und Rechtsvergleichung Universität Konstanz

\title{
Zugang zum Recht - Beobachtungen zur Kostendimension
}

\section{Einführung}

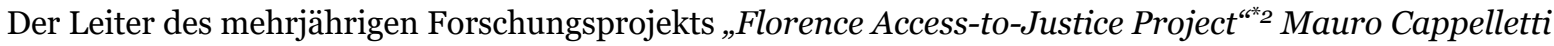
formulierte 1978, dass man sich in einem langen historischen Kampf um den Zugang zum Recht befinde. ${ }^{*}$ Pathetisch klingt es weiter, wenn er erklärt, was damit gemeint ist. So stellt es einen Grundpfeiler eines Rechtssystems dar, dass dieses ,equally accessible to all“ ist. Ein System also, das es dem einzelnen Bürger erlaubt und ermöglicht, seine Rechte zu verteidigen und seine (Rechts-) Streitigkeiten beizulegen, muss für jedermann gleichermaßen zugänglich sein. ${ }^{*} 4$ Auch nach dieser Umschreibung bleibt ein soziologischer ${ }^{*} 5$ Laie zunächst etwas ratlos zurück, was sich im Einzelnen hinter dieser international geführten Debatte verbergen soll. ${ }^{* 6}$ Erst durch die Aufzählung der Barrieren, die einem effektiven Zugang zum Recht entgegenstehen, klärt sich dies auf. ${ }^{*}$ So kann jemand sein Recht oftmals wegen der damit verbundenen Kosten nicht erreichen. ${ }^{* 8}$ Daneben verhindern auch andere Faktoren, dass der Einzelne sein Recht erhält; so z.B. Party Capability $^{* 9}$ und Diffuse Interests ${ }^{*}{ }^{*}$, bekannte Begriffe der internationalen Access to Justice-Debatte. Mit dem Wissen um diese Barrieren benutzt Cappelletti sodann die Wellen-Metapher, um die verschiedenen

1 Die Autorin ist wissenschaftliche Mitarbeiterin am Lehrstuhl für Bürgerliches Recht, Zivilprozessrecht, Internationales Privatrecht und Rechtsvergleichung von Prof. Dr. Astrid Stadler an der Universität Konstanz.

2 Aus dem vierjährigen rechtsvergleichenden und interdisziplinären Forschungsprojekt, das im Herbst 1973 begann und im Jahr 1979 abgeschlossen wurde, resultierte, neben anderen Büchern und Aufsätzen, das 4 Bände umfassende Standardwerk mit dem Namen „Access to Justice“.

3 Mauro Cappelletti, Bryant Garth, 'Access to Justice: The Newest Wave in the Worldwide Movement to Make Rights Effective" (1978) 27 Buff. L. Rev. 181 [182]: „long historical struggle for “access to justice””,

4 Ibid.

5 Genauer ein anthropologischer, ökonomischer, politikwissenschaftlicher und psychologischer Laie, vgl. ibid 181.

6 So auch Matthias Kilian, 'Zugang zum Recht: Beobachtungen in Zeiten von Gesetzen zur Kosteneinsparung bei der Prozesskosten- und Beratungshilfe' [2008] AnwBl 236 [236].

7 Vgl. Mauro Cappelletti, 'Access to Justice: Comparative General Report' (1975) 40 RabelsZ 669 [673ff.]; Mauro Cappelletti, Bryant Garth, "Access to Justice: The Newest Wave in the Worldwide Movement to Make Rights Effective" (1978) 27 Buff. L. Rev. 181 [186ff.].

8 Mauro Cappelletti, 'Access to Justice: Comparative General Report' (1975) 40 RabelsZ 669 [674f.].

9 Ibid 677.

10 Hierzu ibid 680. 
Phasen des „Kampfes“ um einen verbesserten Zugang zum Recht aufzuzeigen und zu identifizieren. ${ }^{*}$ So konzentrierte sich dieser Kampf in einem ersten - im internationalen Vergleich teils unterschiedlich ausgestalteten $^{* 12}$ - Ansatz auf die Etablierung oder den Ausbau der staatlichen Kostenhilfe. Sodann wurde in einer zweiten Welle nach Wegen gesucht, wie dem Problem der Diffuse Interests adäquat begegnet werden kann. ${ }^{*}{ }^{33} \mathrm{Im}$ universalen „Acces-to-Juctice Approach“ schließlich erfolgte die Suche nach Alternativen zur Beilegung von (Rechts-)Streitigkeiten. ${ }^{*} 14$

Im Rahmen dieses Beitrags wird vor dem Hintergrund der ersten Welle die These formuliert, dass sich der deutsche Ansatz zur Verbesserung des Zugangs zum Recht von der staatlichen Finanzierungsintervention in der Form der staatlichen Kostenhilfe zu distanzieren begonnen hat und stattdessen eine vermehrte Hinwendung zur marktbasierten Finanzierungsintervention stattfindet. Um diese These zu überprüfen, soll zunächst die sich in Deutschland sowie Großbritannien vollzogene Entwicklung der staatlichen Kostenhilfe überblicksartig dargestellt werden (dazu 1.). Anschließend sollen Beobachtungen zu den neusten Entwicklungen am deutschen Rechtsdienstleistungsmarkt angestellt werden (dazu 2.). In einem Sonderkapitel gilt es, die dem deutschen Rechtsdienstleistungsmarkt immanenten Regulierungen - mit Blick auf die Ausgestaltung in anderen Mitgliedstaaten der Europäischen Union - näher zu beleuchten, da diese grundsätzlich ein der Kernthese entgegenstehendes Hindernis darstellen (dazu 3.). Zum Schluss soll ein vorsichtiges Resümee hinsichtlich der angestellten Beobachtungen und der sich daraus ergebenden Anforderungen an die Verbesserung des Zugangs zum Recht gewagt werden (dazu 4.).

\section{Entwicklungslinien der staatlichen Kostenhilfe}

\subsection{Aufbau und Abbau der staatlichen Kostenhilfe}

Nach dem Zweiten Weltkrieg ging es auf dem Weg zum deutschen Rechtsstaat zunächst um die Etablierung eines einheitlichen und umfassenden Rechtsschutzsystems über die ordentliche Gerichtsbarkeit hinaus. Erst in den 1970er Jahren richteten sich die deutsche rechtspolitische Debatte und diesbezügliche Reformbestrebungen verstärkt darauf, das vorhandene Rechtsschutzsystem auch denjenigen zugänglich zu machen, die bis dahin nur unzureichend ihre Rechte durchsetzen konnten. ${ }^{*} 15$ Während der in der internationalen rechtsvergleichenden und interdisziplinären Auseinandersetzung so bezeichneten First Wave wurde die Debatte um den Zugang zum Recht anfangs überwiegend auf der Kostenebene geführt. ${ }^{* 16}$ Demnach wurde in Deutschland Ende der 1970er Jahre das Armenrecht zugunsten der Prozesskostenhilfe ${ }^{* 17}$ abgeschafft und die Beratungshilfe ${ }^{*} 18$ eingeführt. ${ }^{*} 19$ Durch eine ungezielte finanzielle Förderung mittels staatlicher Steuergelder sollte das System, welches es den Bürgern ermöglicht, ihre Rechte zu verteidigen und (Rechts-) Streitigkeiten beizulegen, equal accessible to all gemacht werden. ${ }^{* 0}$ Außerdem sollte dieses System so vermehrt Ergebnisse hervorbringen, die individuell und sozial gerecht sind. ${ }^{* 21}$ Dass diese Bestrebungen einen

11 Ibid 681f; Mauro Cappelletti, Bryant Garth, 'Access to Justice: The Newest Wave in the Worldwide Movement to Make Rights Effective" (1978) 27 Buff. L. Rev. 181 [196ff].

12 Vgl. Mauro Cappelletti, 'Access to Justice: Comparative General Report' (1975) 40 RabelsZ 669 [682ff].

13 Ibid 693ff.; vgl. hierzu Astrid Stadler und Hans-W. Micklitz, 'Notwendigkeit eines Verbandsklagegesetzes' in dies. (Hrsg.), Das Verbandsklagerecht in der Informations- und Dienstleistungsgesellschaft (Landwirtschaftsverlag 2005) 1 [1ff].

14 Mauro Cappelletti, 'Access to Justice: Comparative General Report' (1975) 40 RabelsZ 669 [704ff]; Astrid Stadler und HansW. Micklitz, 'Notwendigkeit eines Verbandsklagegesetzes' in dies. (Hrsg.), Das Verbandsklagerecht in der Informations-und Dienstleistungsgesellschaft (Landwirtschaftsverlag 2005) 1 [17].

15 Peter Derleder, 'Gleicher Zugang zum Recht für alle' [2009] VuR 163 [163].

16 Mauro Cappelletti, 'Access to Justice: Comparative General Report' (1975) 40 RabelsZ 669 [682].

17 Gesetz über die Prozesskostenhilfe vom 13.06.1980 (BGBl I, S. 677), in Kraft am 01.01.1981.

18 Gesetz über Rechtsberatung und Vertretung für Bürger mit geringem Einkommen (Beratungshilfegesetz) vom 18.06.1980 (BGBl. I, S. 689), in Kraft am 01.01.1981.

19 Peter Derleder, 'Gleicher Zugang zum Recht für alle' [2009] VuR 163.

20 Vgl. Matthias Kilian, 'Zugang zum Recht: Beobachtungen in Zeiten von Gesetzen zur Kosteneinsparung bei der Prozesskosten- und Beratungshilfe' [2008] AnwBl 236 [236 mwN].

21 Auf diesen zwei Zwecken liegt der Fokus des „access to justice“, vgl. Mauro Cappelletti, Bryant Garth, 'Access to Justice: The Newest Wave in the Worldwide Movement to Make Rights Effective” (1978) 27 Buff. L. Rev. 181 [182]. 
Grundpfeiler eines Rechts- und Sozialstaates darstellen ${ }^{{ }_{2} 2}$, wurde später vom BVerfG ${ }^{{ }_{2} 3}$ unter Berufung auf den Rechtsschutzgedanken des Art. 20 III GG ausdrücklich determiniert. ${ }^{* 24}$ Auf europäischer Ebene ist dieser Grundsatz in Art. 6 EMRK ${ }^{* 25}$ und Art. $47 \mathrm{GRCh}^{* 26}$ abgesichert. Denn wenn das Gewaltmonopol einzig beim Staat liegt und der Einzelne damit zur Durchsetzung seiner Rechte an die Gerichte verwiesen wird, leitet sich daraus die Aufgabe des Staates ab, Gerichte einzurichten und den Zugang zu ihnen jedermann in grundsätzlich gleicher Weise zu eröffnen. ${ }^{* 27}$ Daraus resultiert aber auch, so das BVerfG $1983^{{ }^{*} 28}$, dass ein Bemittelter ebenso wie ein Unbemittelter wirksamen Rechtsschutz in Anspruch nehmen können muss. ${ }^{*}{ }^{29}$

In der internationalen, aber insbesondere in der britischen Debatte zeichnete sich jedoch bereits Mitte der 1990er Jahre immer mehr ab, dass der staatliche Haushalt notorisch schlecht gefüllt ist und die ungezielte finanzielle Förderung staatlicher Kostenhilfe zu derart bescheidenen Ergebnissen bei der Verbesserung des Zugangs zum Recht führte ${ }^{*} 30$, dass die britische Regierung sich 1998 gezwungen sah, den Access to Justice $A c t^{*}{ }^{31} \mathrm{zu}$ erlassen, um künftig bei der staatlichen Kostenhilfe Einsparungen vornehmen zu können. ${ }^{*}{ }^{32}$ Etwas später, im Jahr 2006, gelangte der rechtspolitische Diskurs sodann auch in Deutschland, zu der Auffassung, dass die Aufwendungen für die staatliche Kostenhilfe in der Form der Prozesskosten-, Verfahrenskosten- und Beratungshilfe dringend begrenzt werden müsse. ${ }^{* 33}$ Diesbezügliche im Jahr 2006 beginnende Reformbestrebungen fußten auf dem Verdikt, dass Deutschland allein in diesem Jahr für die Prozesskostenhilfe in Zivilsachen 490 Mio. Euro aufgewandt hatte und diese Tendenz steigend war. Dies entsprach 5,95 Euro für die Prozesskostenhilfe pro Bürger. ${ }^{*} 34$ Beim Allzeithoch im Jahr 2008 betrugen die Aufwendungen für die Prozesskostenhilfe in der ordentlichen Gerichtsbarkeit sowie für die Beratungshilfe in der Summe sogar 589 Mio. Euro. ${ }^{*} 35$ Dass Deutschland im internationalen Vergleich seit jeher vergleichsweise wenig für die staatliche Kostenhilfe ausgibt, blieb damals in der rechtspolitischen Debatte oftmals außen vor. So sind die Pro-Kopf-Ausgaben für staatliche Kostenhilfe in den Niederlanden, Schweden und Norwegen fünfmal, in Großbritannien sogar zehnmal so hoch. ${ }^{*}{ }^{6}$ Den Anstoß zur Reform lieferten damals

22 Peter Derleder, 'Gleicher Zugang zum Recht für alle’ [2009] VuR 163 [164]; Matthias Kilian, 'Gedanken zur Kostenrechtsmodernisierung II: Prozesskosten und Beratungshilfe - Den Zugang zum Recht sichern: Tatsachen, Anforderungen, Probleme und Lösungsideen' [2014] AnwBl 46 [46].

23 BVerfGE 81, 347 [356]; NJW 1991, 413 [414].

24 Schon viel früher wurde aus dem Sozialstaatsprinzip und dem allgemeinen Gleichheitssatz die Forderung nach einer „weitgehenden Angleichung der Situation von Bemittelten und Unbemittelten im Bereich des Rechtsschutzes“ abgeleitet. Vgl. Peter Derleder, 'Gleicher Zugang zum Recht für alle' [2009] VuR 163 [164]; BVerfGE 9, 124 [130f]; NJW 1959, 715 [716].

25 EGMR, Urteil vom 15.02.2005 - 68416/01, Steel und Morris/Vereinigtes Königreich; EGMR (V. Sektion), Entscheidung vom 08.12.2009 - 54193/07 Herma/Deutschland.

26 EuGH, Urteil vom 22.12.2010 - C-279/09, DEB Deutsche Energiehandels- und Beratungsgesellschaft mbH/Deutschland.

27 BVerfGE 81, 347 [356]; NJW 1991, 413 [414]; vgl. hierzu auch, Entwurf eines Gesetzes zur Begrenzung der Aufwendungen für die Prozesskostenhilfe (Prozesskostenhilfebegrenzungsgesetz - PKHBegrenzG), BT-Drucks. 16/1994 S. 1 und 12: https://www.neuerichter.de/fileadmin/user_upload/lv_berlin_brandenburg/BT-Drs_16_1994.pdf; zuletzt abgerufen am 14.01.2021.

28 BVerfGE 63, 380 [395]; NJW 1983, 1599 [1600].

29 Vgl. Peter Derleder, 'Gleicher Zugang zum Recht für alle’ [2009] VuR 163 [164].

30 Matthias Kilian, 'Zugang zum Recht: Beobachtungen in Zeiten von Gesetzen zur Kosteneinsparung bei der Prozesskostenund Beratungshilfe' [2008] AnwBl 236 [236].

31 https://www.legislation.gov.uk/ukpga/1999/22/contents; zuletzt abgerufen am 14.01.2021.

32 Matthias Kilian, 'Zugang zum Recht: Beobachtungen in Zeiten von Gesetzen zur Kosteneinsparung bei der Prozesskostenund Beratungshilfe' [2008] AnwBl 236 [239].

33 Vgl. Entwurf eines Gesetzes zur Begrenzung der Aufwendungen für die Prozesskostenhilfe (Prozesskostenhilfebegrenzungsgesetz - PKHBegrenzG), BT-Drucks. 16/1994 - http://dip21.bundestag.de/dip21/btd/16/019/1601994.pdf; zuletzt abgerufen am 14.01.2021; Entwurf eines Gesetzes zur Änderung des Beratungshilferechts, BR-Drucks. 648/08 -http://dipbt. bundestag.de/dip21/brd/2008/0648-08.pdf; zuletzt abgerufen am 14.01.2021; zudem sollten bereits mit einem Gesetzentwurf von 2004 Gerichtsgebühren für das Sozialgerichtsverfahren eingeführt werden, Entwurf eines Gesetzes zur Änderung des Sozialgerichtsgesetzes, BR-Drucks. 663/03 - http://dipbt.bundestag.de/dip21/brd/2003/0663-03.pdf; zuletzt abgerufen am 14.01.2021; Peter Derleder, 'Gleicher Zugang zum Recht für alle' [2009] VuR 163 [164ff].

34 Matthias Kilian, 'Zugang zum Recht: Beobachtungen in Zeiten von Gesetzen zur Kosteneinsparung bei der Prozesskostenund Beratungshilfe' [2008] AnwBl 236 [240].

35 Matthias Kilian, 'Gedanken zur Kostenrechtsmodernisierung II: Prozesskosten und Beratungshilfe - Den Zugang zum Recht sichern: Tatsachen, Anforderungen, Probleme und Lösungsideen' [2014] AnwBl 46 [46].

36 Matthias Kilian, 'Zugang zum Recht: Beobachtungen in Zeiten von Gesetzen zur Kosteneinsparung bei der Prozesskosten- und Beratungshilfe' [2008] AnwBl 236 [240]; Matthias Kilian, 'Gedanken zur Kostenrechtsmodernisierung II: Prozesskosten und Beratungshilfe - Den Zugang zum Recht sichern: Tatsachen, Anforderungen, Probleme und Lösungsideen’ [2014] AnwBl 46 [47]. 
die Länder Baden-Württemberg und Niedersachsen. Als schlagendes Argument wurde dabei das bundesweite Einsparungspotenzial von ca. 100 Mio. Euro genannt. ${ }^{*} 37$ Angesichts der weltweiten Finanzkrise im Jahr 2008/2009, in der Konjunkturprogramme in enormen Summen nötig und von staatlicher Seite bewilligt wurden, schien eine Kürzung der überschaubaren Aufwendungen für die Gewährung gleichen Zugangs zum Recht am Ende politisch nicht tragbar. ${ }^{*} 38$ Die Reformbestrebungen scheiterten schlussendlich im Februar 2009 am Veto der SPD-Fraktion im Deutschen Bundestag. ${ }^{*}{ }^{9}$ Schon damals war aber absehbar, dass von politischer Seite eine Einsparung bei der Beratungs-, Verfahrenskosten- und Prozesskostenhilfe auch weiterhin als erforderlich angesehen würde. ${ }^{*}{ }^{40}$ So reichten dann auch die Länder die bereits in der 16. Legislaturperiode eingebrachten Reformgesetzentwürfe ${ }^{* 11}$ erneut 2012 - mit teilweise veraltetem Zahlenmaterial in der Gesetzesbegründung - in der 17. Legislaturperiode ein. ${ }^{*}{ }^{42}$ Dass zu diesem Zeitpunkt die Aufwendungen für die Kostenhilfe bereits wieder rückläufig waren, konnte somit nicht mehr in Ansatz gebracht werden. ${ }^{*} 43$ Ebenso wenig konnten die aus Rückflüssen resultierenden Einnahmen - zumindest noch im Gesetzentwurf des Bundesrates - beziffert werden. Ausgaben für die staatliche Kostenhilfe wurden dort schlicht mit deren Kosten gleichgesetzt. ${ }^{*} 44$ Zwar gab es auch Reformziele, die durchaus sinnvoll waren, so insbesondere die Bekämpfung des Missbrauchs der Prozesskostenhilfe durch mutwillige Rechtsverfolgung. ${ }^{*} 45$ Mit dem am 01. Januar 2014 in Kraft getretenen Gesetz zur Änderung des Prozesskostenhilfe- und Beratungshilferechts ${ }^{*} 46$ distanzierte man sich jedoch vom Ansatz der staatlichen Finanzierungsintervention zur Verbesserung des Zugangs zum Recht.

\subsection{Ergründung neuer Wege bei der Verbesserung des Zugangs zum Recht durch eingehende Bedürfnisidentifizierung}

\subsection{1. Überblick über den angelsächsischen Ansatz der Bedürfnisidentifizierung}

Dass diese Abkehr nicht zwangsläufig als politische Herabstufung der Bedeutung des Prinzips der Gleichheit vor dem Recht aufgefasst werden muss ${ }^{*} 47$, zeigt die Mitte der 1990er Jahre voranschreitende „ReEngineering "**48-Entwicklung in England. Dort wurde nach dem gescheiterten Ansatz der staatlichen Finanzierungsintervention intensiv nach neuen Ansätzen zur Verbesserung des Zugangs zum Recht gesucht. Dabei lag der Fokus zunächst auf der Bedürfnisidentifizierung der rechtssuchenden Bevölkerung. ${ }^{*} 49$

37 Peter Derleder, 'Gleicher Zugang zum Recht für alle' [2009] VuR 163 [164].

38 Peter Derleder, 'Gleicher Zugang zum Recht für alle’ [2009] VuR 163 [165].

39 Vgl. Peter Derleder, ‘Gleicher Zugang zum Recht für alle’ [2009] VuR 163 [164].

40 So schon Peter Derleder, 'Gleicher Zugang zum Recht für alle’ [2009] VuR 163 [168].

41 Prozesskostenhilfe: BT-Drucks. 16/1994 - http://dip21.bundestag.de/dip21/btd/16/019/1601994.pdf; zuletzt abgerufen am 14.01.2021; 17/1216 - https://dip21.bundestag.de/dip21/btd/17/012/1701216.pdf; zuletzt abgerufen am 14.01.2021; Beratungshilfe: BT-Drucks. 17/2164 - https://dip21.bundestag.de/dip21/btd/17/021/1702164.pdf; zuletzt abgerufen am 14.01.2021.

42 Entwurf eines Gesetzes zur Änderung des Prozesskostenhilfe- und Beratungshilferechts, BR-Drucks. 516/12 - https://www. bundesrat.de/SharedDocs/drucksachen/2012/0501-0600/516-12.pdf?__blob=publicationFile\&v=3; zuletzt abgerufen am 14.01.2021.

43 Vgl. Matthias Kilian, ‘Gedanken zur Kostenrechtsmodernisierung II: Prozesskosten und Beratungshilfe - Den Zugang zum Recht sichern: Tatsachen, Anforderungen, Probleme und Lösungsideen’ [2014] AnwBl 46 [46, 48].

44 Erst im Gesetzentwurf der Bundesregierung BT-Drucks. 17/11472 ist dieses Problem erkannt und teilweise behoben worden, vgl. Matthias Kilian, 'Gedanken zur Kostenrechtsmodernisierung II: Prozesskosten und Beratungshilfe - Den Zugang zum Recht sichern: Tatsachen, Anforderungen, Probleme und Lösungsideen' [2014] AnwBl 46 [48]; Matthias Kilian, 'Zugang zum Recht: Beobachtungen in Zeiten von Gesetzen zur Kosteneinsparung bei der Prozesskosten- und Beratungshilfe' [2008] AnwBl 236 [240].

45 Vgl. hierzu bereits Peter Derleder, 'Gleicher Zugang zum Recht für alle' [2009] VuR 163 [164f]; Matthias Kilian, 'Gedanken zur Kostenrechtsmodernisierung II: Prozesskosten und Beratungshilfe - Den Zugang zum Recht sichern: Tatsachen, Anforderungen, Probleme und Lösungsideen' [2014] AnwBl 46 [48].

46 Vom 31.08.2013 (BGBl I, S. 3533).

47 So auch Matthias Kilian, 'Zugang zum Recht: Beobachtungen in Zeiten von Gesetzen zur Kosteneinsparung bei der Prozesskosten- und Beratungshilfe' [2008] AnwBl 236 [239].

48 Vgl. Richard Moorhead and Pascoe Pleasence, After Universalism: Re-engineering Access to Justice (Wiley-Blackwell 2003).

49 Matthias Kilian, 'Zugang zum Recht: Beobachtungen in Zeiten von Gesetzen zur Kosteneinsparung bei der Prozesskostenund Beratungshilfe' [2008] AnwBl 236 [236f mwN]. 
Entsprechende empirische Erkenntnisse, die überhaupt erst viele neue Lösungsansätze ermöglichten ${ }^{*} 50$, verdeutlichten, dass es neben der Kostendimension oftmals an dem Wissen der rechtssuchenden Bevölkerung fehlt, an wen sie sich wenden sollen und wie ein Problemlösungsprozess initiiert werden kann. Besonders häufig verfielen dabei Personen mit niedrigem Einkommen und Bildungsmilieu und damit aufgrund dieser besonderen Verletzlichkeit benachteiligte Bevölkerungsgruppen in eine Lethargie, ihre Rechtsprobleme dem Zugang zum Recht zuzuführen. ${ }^{*}{ }^{51}$ Zudem kristallisierte sich in der internationalen Access to Justice-Debatte immer mehr heraus, dass sich rechtssuchende Bürger oftmals einem Lebensproblem ausgesetzt sehen, das nur bedingt einen juristischen Kern hat. Eine frühzeitige Eliminierung dieser sog. trigger problems kann dazu führen, dass sich die rechtlichen Bedürfnisse der Bevölkerung reduzieren, da es nicht zu weitreichenden, auch die Kostenhilfe beanspruchenden Folgeproblemen kommen kann. Damit verringern sich nicht nur die Kosten für die Volkswirtschaft und den Sozialstaat, sondern es verbessert sich auch die Lebensqualität der Bürger. ${ }^{* 2}$ Dass insbesondere sog. additive Effekte eines nicht gelösten Rechtsproblems erhebliche mittelbare wie unmittelbare negative volkswirtschaftliche und damit ökonomische Nebeneffekte hervorrufen können, wurde im Zuge von breit angelegten Bevölkerungsumfragen ebenfalls erforscht und empirisch belegt. So gaben viele Befragte an, dass sie aufgrund eines Rechtsproblems Schwierigkeiten hatten, ihr Leben normal zu führen, was in Problemen bei der Erwerbstätigkeit sowie beim Einkommen resultierte. ${ }^{*} 3$

\subsubsection{Erforschung der rechtlichen Bedürfnisse der Bevölkerung in Deutschland}

Fraglich ist, ob eine ähnlich intensive Bedürfnisidentifizierung auch in Deutschland vor oder nach der Änderung des Prozesskostenhilfe- und Beratungshilferechts stattgefunden hat bzw. stattfindet. Auch auf dem Weg zu einer kostengünstigeren Verbesserung beim deutschen Zugang zum Recht wäre eine - über die Kostendimension hinausgehende - empirische Ermittlung der Bedürfnisse der Bevölkerung unerlässlich. Anders als im internationalen, insbesondere im angelsächsischen Bereich lässt sich jedoch in Deutschland-- soweit ersichtlich - nur vereinzelt systematische empirische Forschung, die sich mit den unmet legal needs ${ }^{*} 54$ der Bürger befasst, erkennen. ${ }^{*} 55$ So ergab z.B. eine umfassende Bevölkerungsumfrage, „Mandanten und ihre Anwälte“, des Soldan Instituts im Zeitraum von 2006 bis $2007^{*}{ }^{56}$, dass insbesondere die Möglichkeit zu einem sofortigen Gespräch mit einem Rechtsanwalt wie auch die Möglichkeit, in nächster Zeit einen Termin zu erhalten, für über $83 \%$ der Befragten wichtig bis sehr wichtig ist. ${ }^{*} 57$ Auch wenn es sich hierbei nur um vereinzelte und keine unabhängigen, flächendeckenden sowie vom Staat initiierten empi-

50 So wurde z.B. eine Art Empfehlungsnetzwerk, als ,first point of call“, in der Form von staatlichen Beratungseinrichtungen, im Zuge der ,Re-Enginiering“-Entwicklung, durch den Community Legal Service eingerichtet, die die bestehenden Angebote besser miteinander vernetzt und dadurch zugänglich macht. Matthias Kilian, 'Zugang zum Recht: Beobachtungen in Zeiten von Gesetzen zur Kosteneinsparung bei der Prozesskosten- und Beratungshilfe' [2008] AnwBl 236 [238f mwN].

51 Ibid 237ff; Matthias Kilian, ‘Gedanken zur Kostenrechtsmodernisierung II: Prozesskosten und Beratungshilfe - Den Zugang zum Recht sichern: Tatsachen, Anforderungen, Probleme und Lösungsideen' [2014] AnwBl 46 [48].

52 Matthias Kilian, 'Zugang zum Recht: Beobachtungen in Zeiten von Gesetzen zur Kosteneinsparung bei der Prozesskosten- und Beratungshilfe' [2008] AnwBl 236 [238]; Matthias Kilian, 'Gedanken zur Kostenrechtsmodernisierung II: Prozesskosten und Beratungshilfe - Den Zugang zum Recht sichern: Tatsachen, Anforderungen, Probleme und Lösungsideen’ [2014] AnwBl 46 [48f].

53 Matthias Kilian, 'Zugang zum Recht: Beobachtungen in Zeiten von Gesetzen zur Kosteneinsparung bei der Prozesskosten- und Beratungshilfe' [2008] AnwBl 236 [238 mwN]; Matthias Kilian, 'Gedanken zur Kostenrechtsmodernisierung II: Prozesskosten und Beratungshilfe - Den Zugang zum Recht sichern: Tatsachen, Anforderungen, Probleme und Lösungsideen' [2014] AnwBl 46 [49 mwN].

54 Zum Begriff Mauro Cappelletti, 'Access to Justice: Comparative General Report' (1975) 40 RabelsZ 669 [678].

55 Matthias Kilian, 'Zugang zum Recht: Beobachtungen in Zeiten von Gesetzen zur Kosteneinsparung bei der Prozesskostenund Beratungshilfe' [2008] AnwBl 236 [237].

56 Vgl. Christoph Hommerich, Matthias Kilian, Mandanten und ihre Anwälte: Ergebnisse einer Bevölkerungsumfrage zur Inanspruchnahme und Bewertung von Rechtsdienstleistungen, (DeutscherAnwaltVerlag 2007); Christoph Hommerich, Matthias Kilian, 'Warum Bürger keinen Anwalt beauftragen - Wie Rechtsprobleme ohne Anwalt gelöst werden' [2007] AnwBl 783.

57 Vgl. Christoph Hommerich, Matthias Kilian,'Die Auswahl von Experten durch Laien - Worauf Bürger achten, wenn sie einen Rechtsanwalt suchen' [2007] AnwBl 858; Matthias Kilian, ‘Gedanken zur Kostenrechtsmodernisierung II: Prozesskosten und Beratungshilfe - Den Zugang zum Recht sichern: Tatsachen, Anforderungen, Probleme und Lösungsideen' [2014] AnwBl 46 [50]; Christoph Hommerich, Matthias Kilian, 'Warum Bürger keinen Anwalt beauftragen - Wie Rechtsprobleme ohne Anwalt gelöst werden’ [2007] AnwBl 783 [784]. 
rischen Forschungen handelt, zeichnet sich die Tendenz ab, dass es durch den Verrechtlichungsprozess der letzten Jahrzehnte ${ }^{*} 58$ für die rechtssuchende Bevölkerung - insbesondere für Angehörige niedriger Bildungsmilieus ${ }^{*} 59$ - ein Bedürfnis von überragender Wichtigkeit darstellt, beim Auftreten eines Rechtsproblems schnell und unkompliziert Zugang zu einem fachkundigen Dritten zu erhalten. Dabei ist es für den Rechtssuchenden oftmals von größerer Wichtigkeit, schnell eine Auskunft zu erhalten, als etwa die Fachkompetenz oder die örtliche Nähe des Ratgebers. ${ }^{*} 60$

Auch im Hinblick auf die Kostendimension lassen sich nur vereinzelt - überwiegend für die Versicherungsbranche - erhobene empirische Studien anführen. ${ }^{* 61}$ Der ROLAND Rechtsreport von 2020 ermittelte die öffentliche Meinung zum deutschen Rechtssystem und anderen ausgewählten rechtspolitischen Schwerpunktthemen für die ROLAND Rechtsschutz-Versicherungs-AG. Durchgeführt wurde die statistische Erhebung vom Institut für Demoskopie Allensbach (IfD), das für 20201.228 Personen befragte. ${ }^{* 62}$ Es zeigte sich, dass Bürger im Durchschnitt erst bei einem Streitwert von 1.840 Euro gerichtlichen Rechtsschutz suchen. ${ }^{*} 63$ Daneben gaben etwa $25 \%$ der Befragten an, in den vergangenen fünf Jahren zwar einen Bedarf nach rechtlichem Beistand gehabt, diesen jedoch bewusst nicht ergriffen zu haben. ${ }^{*} 64$ Laut einer repräsentativen forsa-Studie aus dem Jahr 2013, die im Auftrag des Gesamtverbandes der Deutschen Versicherungswirtschaft (GDV) durchgeführt wurde, scheuten $71 \%$ der Befragten vor der Beauftragung eines Rechtsanwalts aufgrund der Kosten zurück. ${ }^{*}{ }^{6}$ Neben dem verfassungsrechtlich abgesicherten oben genannten Prinzip, dass der Zugang zum Recht nicht aufgrund wirtschaftlicher Leistungsfähigkeit versperrt sein darf ${ }^{* 66}$, ergibt sich dabei aus dem Justizgewährungsanspruch, der aus dem Rechtsstaatsprinzip Art. 20 III des Grundgesetz folgt, dass das Kostenrisiko nicht zu dem mit dem Verfahren angestrebten wirtschaftlichen Erfolg außer Verhältnis stehen darf, sodass die Anrufung der Gerichte nichtmehr sinnvoll erscheint. ${ }^{*} 7$ Ebenso ist dieses Prinzip in Art. 6 EMRK und Protokoll Nr. 1 Art. 1 der EMRK verbürgt. ${ }^{* 68}$ Unter Berücksichtigung dieser Bedürfnisse der rechtssuchenden Bevölkerung lassen sich Beobachtungen der neusten Entwicklungen auf dem Rechtsdienstleistungsmarkt leichter darstellen.

58 Peter Derleder, 'Gleicher Zugang zum Recht für alle’ [2009] VuR 163 [163 mwN].

59 Christoph Hommerich, Matthias Kilian, 'Warum Bürger keinen Anwalt beauftragen - Wie Rechtsprobleme ohne Anwalt gelöst werden' [2007] AnwBl 783 [784].

60 Christoph Hommerich, Matthias Kilian, Mandanten und ihre Anwälte: Ergebnisse einer Bevölkerungsumfrage zur Inanspruchnahme und Bewertung von Rechtsdienstleistungen, (DeutscherAnwaltVerlag 2007) 110; Matthias Kilian, 'Gedanken zur Kostenrechtsmodernisierung II: Prozesskosten und Beratungshilfe - Den Zugang zum Recht sichern: Tatsachen, Anforderungen, Probleme und Lösungsideen' [2014] AnwBl 46 [50].

61 So insb. die seit 10 Jahren jährlich von dem Institut für Demoskopie Allensbach für die ROLAND Rechtsschutz-VersicherungsAG durchgeführten Rechtsreporte. Rechtsreport von 2020 - https://www.ifd-allensbach.de/fileadmin/IfD/sonstige_pdfs/ ROLAND_Rechtsreport_2020.pdf; zuletzt abgerufen am 14.01.2021.

62 Dass sich Gesetzesentwurfsbegründungen trotz dieser marginalen Anzahl an Befragten, diesen Umfragen zur Darstellung bedienen, könnte als Indiz dafür gewertet werden, dass es an umfassenderem statistischen Datenmaterial fehlt, vgl. Entwurf eines Gesetzes zur Förderung verbrauchergerechter Angebote im Rechtsdienstleistungsmarkt vom 12.11.2020 - https://www. bmjv.de/SharedDocs/Gesetzgebungsverfahren/Dokumente/RefE_Rechtsdienstleister.pdf?_blob=publicationFile\&v=1, S. 10; zuletzt abgerufen am 14.01.2021.

63 Vgl. ROLAND Rechtsreport 2020 - Einstellung der Bevölkerung zum deutschen Justizsystem und zur außergerichtlichen Konfliktlösung, S. 24, Schaubild 15; - https://www.ifd-allensbach.de/fileadmin/IfD/sonstige_pdfs/ROLAND_Rechtsreport_2020.pdf; zuletzt abgerufen am 14.01.2021.

64 Vgl. ROLAND Rechtsreport 2020 - Einstellung der Bevölkerung zum deutschen Justizsystem und zur außergerichtlichen Konfliktlösung, S. 23, Schaubild 14; - https://www.ifd-allensbach.de/fileadmin/IfD/sonstige_pdfs/ROLAND_Rechtsreport_2020.pdf; zuletzt abgerufen am 14.01.2021; Eine aus dem Jahr 2010 durchgeführte Consumer Empowerment Survey kam für europäische Verbraucher dabei zu ähnlichen Ergebnissen auf europäischer Ebene. So würde jeder zweite Verbraucher, bei einem Streitwert bis zu 500 Euro keine gerichtliche Klage erheben, wo hingegeben 20\% davon ausgingen, dass ein Anlass zur Beschwerde bezüglich einer Verbraucherkauftransaktion bestand, vgl. Consumer Empowerment Survey, Eurobarometer Nr. 342, 2010, S. 217 und S. 169 - https://ec.europa.eu/commfrontoffice/publicopinion/archives/ebs/ ebs_342_en.pdf; zuletzt abgerufen am 14.01.2021.

65 Vgl. Ängste und Erwartungen von Verbrauchern bei rechtlichen Auseinandersetzungen, S. 10 - https://www.gdv.de/ resource/blob/30990/916cec00467920d4121f3f234e109ba5/studie-forsa-studie-kosten-eines-rechtsstreits-data.pdf; zuletzt abgerufen am 14.01.2021.

66 Vgl. oben Fn 23.

67 BVerfGE 85, 337; NJW 1992, 344.

68 BVerfGE 107, 395 [406f], NJW 2003, 1102; Max Vollkommer in Richard Zöller (Hrsg.), ZPO (33. Auflage Otto Schmidt) Einleitung Rn. $48 \mathrm{mwN}$. 


\section{Beobachtungen der neusten Entwicklungen am deutschen Rechtsdienstleistungsmarkt}

Wie bereits angesprochen kam schon 2013 eine forsa-Studie im Auftrag des GDV zu dem Schluss, dass nach wie vor ca. 70\% der Bevölkerung durch die Kosten, die eine anwaltliche Rechtsauskunft und -beratung verursacht, davon abgeschreckt werden, sich rechtlichen Rat einzuholen. ${ }^{* 69}$ Dieser Befund sowie die anderen oben genannten empirischen Erforschungen suggerieren, dass ein Bedürfnis nach schneller, effektiver und kostengünstiger Rechtsdienstleistung im Sinne der Rechtsauskunft und -beratung bei der Bevölkerung und insbesondere bei Verbrauchern besteht, der Anwalt aber nicht die präferierte Anlaufstelle darstellt. ${ }^{*} 70$ Die Befriedigung des Bedürfnisses nach Rechtsdienstleistungen im Sinne einer Rechtsauskunft und -beratung, ob eventuelle Ansprüche dem Grunde nach tatsächlich bestehen, generiert sodann bei der Bejahung dieser Frage folgelogisch ein neues Bedürfnis, nämlich das nach schneller, effektiver und kostengünstiger Rechtsdurchsetzung. Denn oftmals hindert ein rationales Desinteresse die eigenverantwortliche und individuelle Rechtsdurchsetzung der Ansprüche, da aus der Sicht des Anspruchsberechtigten und potenziellen Klägers die Kosten der Rechtsverfolgung und das oftmals anschließende Risiko des Prozessverlusts die Wahrscheinlichkeit der erfolgreichen Rechtsdurchsetzung sowie des Prozessgewinns überwiegen, und es wird sowohl von der Rechtsverfolgung als auch von einer Klageerhebung Abstand genommen. ${ }^{*}{ }^{71}$ Dieses rationale Desinteresse wirkt sich dahingehend negativ aus, dass das Schadensrecht seine verhaltenssteuernde Funktion für die Zukunft verliert. ${ }^{*}{ }^{2}$ Dem ökonomischen Geist entsprechend wird zudem am Markt immer dort, wo Bedürfnisse bestehen und somit ein Mangel vorliegt, zur Befriedigung dieser Bedürfnisse gewirtschaftet und es werden auf ökonomische Weise Güter und Dienstleistungen bereitgestellt. ${ }^{*} 73$

So ist es dann auch wenig verwunderlich, dass sich in den letzten vier bis fünf Jahren auf dem deutschen Rechtsdienstleistungsmarkt verstärkt Geschäftsmodelle etabliert haben, die Dienstleistungen zur Befriedigung nicht nur eines der zuvor genannten Bedürfnisse, sondern zur Befriedigung beider Bedürfnisse anbieten. ${ }^{*} 74 \mathrm{Im}$ Grunde handelt es sich bei den verschiedensten am Markt auftretenden Anbietern, z.B. „flightright“* ${ }^{\star *} 75$ und „wenigermiete.de ${ }^{\star *} 76$, um im Kern ähnliche Geschäftsmodelle. Überwiegend werden dabei unter Zuhilfenahme softwarebasierter Möglichkeiten und damit weitestgehend automatisierter

69 Vgl. Ängste und Erwartungen von Verbrauchern bei rechtlichen Auseinandersetzungen, S. 10 - https://www.gdv.de/ resource/blob/30990/916cec00467920d4121f3f234e109ba5/studie-forsa-studie-kosten-eines-rechtsstreits-data.pdf; zuletzt abgerufen am 14.01.2021.

70 So auch, Markus, Hartung, 'Inkasso, Prozessfinanzierung und das RDG - Was darf ein Legal-Tech-Unternehmen als Inkassodienstleister?” [2019] AnwBl 353 [354]; vgl. ferner Volker Römermann, 'Tore auf für Legal Tech' (LTO, 12.12.2019) https://www.lto.de/recht/juristen/b/legal-tech-bgh-lexfox-wenigermiete-berufsrecht-rdg-anwaelte-erfolgshonorar/; zuletzt abgerufen am 14.01.2021.

71 Zum rationalen Desinteresse in Bezug auf den kollektiven Rechtsschutz vgl. Hans-Bernd Schäfer, 'Anreizwirkungen bei der Class Action und der Verbandsklage' in Jürgen Basedow, Klaus J Hopt, Hein Kötz und Dietmar Baetge (Hrsg.), Bündelung gleichgerichteter Interessen im Prozess (Mohr Siebeck 1999) 67ff; Markus Hartung, 'Legal Tech und das RDG - Raus aus der Beziehungskrise! - Warum es bei der Legal Tech-Diskussion nicht um Tech, sondern um den Zugang zum Recht geht' [2020] AnwBl 8.

72 Darauf kann an dieser Stelle nicht näher eingegangen werden vgl. aber eingehend dazu, Hans-Bernd Schäfer, 'Anreizwirkungen bei der Class Action und der Verbandsklage’ in Jürgen Basedow, Klaus J Hopt, Hein Kötz und Dietmar Baetge (Hrsg.), Bündelung gleichgerichteter Interessen im Prozess (Mohr Siebeck 1999) 67 [68f].

73 Vgl. Hans-Bernd Schäfer, Claus Ott, Lehrbuch der ökonomischen Analyse des Zivilrechts (5. Auflage, Springer 2012) XXXIII. - DOI: https://doi.org/10.1007/978-3-642-29122-7.

74 Vorreiter war hier Flightright - https://www.flightright.de/, die die pauschalisierten Schadensersatzansprüche die den Fluggästen gem. FluggastrechteVO (EG) Nr. 261/2004 des europäischen Parlaments und des Rates vom 11.02.2004 zustehen, schnell und kostengünstig realisieren; vgl. Markus Hartung, 'Legal Tech und das RDG - Raus aus der Beziehungskrise! Warum es bei der Legal Tech-Diskussion nicht um Tech, sondern um den Zugang zum Recht geht' [2020] AnwBl 8 [8f]; vgl. zu dem Geschäftsmodell von myRight, Alexander Morell, 'Keine Kooperation ohne Konflikt' [2019] JZ 809. - DOI: https:// doi.org/10.1628/jz-2019-0299.

75 https://www.flightright.de/.

76 https://www.wenigermiete.de/, bietet auf ihrer Plattform z.B. eine Funktion an, mittels derer sich nach Eingabe gewisser Rahmendaten bezüglich des Mietobjekts, eine erste Einschätzung zum Sparpotenzial bezüglich des Mietzinses darstellen lassen soll. Grundsätzlich erwartet den Rechtssuchenden jedoch zunächst lediglich die Auskunft, dass sich der Fall zur Rechtsdurchsetzung anbietet oder nicht anbietet sowie im ersten Fall, die damit verbundene Möglichkeit einen Auftrag zu erteilen, dass der Einzelfall durch einen Experten geprüft werden soll, vgl. Schriftliche Stellungnahme von Prof. Dr. Christian Wolf zu dem Gesetzentwurf - https://brak.de/w/files/04_fuer_journalisten/presseerklaerungen/2020_03_10_ipa-stn.zu-gesetz-ee_rdg-modernsg_bt-drs.19_9527u.19_16884.pdf S. 7f; zuletzt abgerufen am 14.01.2021. 
Vorgänge (Legal-Tech-Anwendungen) ${ }^{*} 77$ auf den Plattformen der Anbieter zunächst Tools und Eingabemasken ${ }^{*} 78$ zur Einschätzung angeboten, ob ein vermeintlicher Anspruch zur außergerichtlichen oder gerichtlichen Anspruchsdurchsetzung dem Grunde nach besteht. ${ }^{*} 9$ Ist dies zu bejahen, obliegt es dem Gläubiger dieser Ansprüche, den Dienstleister mit der Anspruchsdurchsetzung zu beauftragen. ${ }^{*} 80$ Dies geschieht überwiegend, indem die Ansprüche an den Dienstleister zur treuhänderischen Einziehung abgetreten werden (sog. Inkassozession). ${ }^{*}{ }^{11}$ Sodann erfolgt eine zunächst außergerichtliche und im Falle der Erfolglosigkeit der außergerichtlichen Bemühungen eine gerichtliche Anspruchsdurchsetzung für den Kunden. Das Besondere an derartigen Geschäftsmodellen und der Grund, weswegen sich die Rechtssuchenden zur Bedürfnisbefriedigung im großen Stil dieser Unternehmen bedienen ${ }^{*}{ }^{2}$, ist, dass sie zusätzlich zur schnellen und unkomplizierten (Erst-)Einschätzung das rationale Desinteresse an einer Anspruchsdurchsetzung dahingehend fast vollständig eliminieren, dass diese Unternehmen neben der völligen Konfliktdelegation - der Kunde wird durch die Abtretung seiner Ansprüche komplett aus der Eigenverantwortung entlassen ${ }^{*} 3$ - auch die Kosten von der außergerichtlichen bis hin zur gerichtlichen Geltendmachung bei erfolgloser Rechtsdurchsetzung übernehmen. ${ }^{*} 4$ Man kann diesen Geschäftsmodellen, die diese Unternehmen anbieten, im Einzelnen kritisch gegenüberstehen. ${ }^{*} 5$ Zugutezuhalten ist ihnen jedoch, dass diese Dienstleistung eine große Anreizwirkung auf die traditionell apathischen und eigenverantwortungsscheuenden Rechtssuchenden auch oberhalb der Berechtigung zur staatlichen Kostenhilfe ausübt. Dieser Anreiz wird auch nicht dahingehend geschmälert, dass als einziger (offensichtlicher) ${ }^{*} 86$ Haken an der Sache, der Unternehmer im Fall der erfolgreichen Geltendmachung der Ansprüche einen Teil der ausgekehrten Schadens- bzw. Erlössumme einbehält. ${ }^{*} 87$ Das „Rundumsorglospaket ${ }^{* * 88}$ hat einen stattlichen Preis, den der Adressatenkreis zur Bedürfnisbefriedigung jedoch zu zahlen bereit ist.

Im Allgemeinen kann festgehalten werden, dass jedes zusätzliche Hilfsangebot respektive (Geschäfts-) Modell, sei es nun staatlicher oder marktbasierter Natur, das den Zugang zum Recht verbessert, einen Gewinn für den Rechtssuchenden und damit für den Rechtsstaat darstellt. Denn wenn eine der Barrieren,

77 Vgl. zur Begriffsdefinition, Christina-Maria Leeb, Digitalisierung, Legal Technology und Innovation (Duncker\&Humblot 2019) 49ff. - DOI: https://doi.org/10.3790/978-3-428-55784-4; Michael Grupp, 'Legal Tech - Impulse für Streitbeilegung und Rechtsdienstleistung - Informationstechnologische Entwicklung an der Schnittstelle von Recht und IT' [2014] AnwBl 660; Martin Fries, 'Staatsexamen für Roboteranwälte? - Optionen für die Regulierung von Legal-Tech-Dienstleister' [2018] ZRP 161.

78 Fabian Widder, 'Verbraucherschutz und RDG - wo bleibt die Anwaltschaft? - Legal Tech - Notwendigkeit einer gesetzlichen Regelung nach dem BGH-Urteil Lexfox' [2020] AnwBl 269.

79 Markus Hartung, 'Legal Tech und das RDG - Raus aus der Beziehungskrise! - Warum es bei der Legal Tech-Diskussion nicht um Tech, sondern um den Zugang zum Recht geht' [2020] AnwBl 8, ein Befürworter dieser Geschäftsmodelle, kommt dennoch zu der Ansicht, dass es sich bei dieser Art der Rechtsdienstleistung in der Form der Rechtsberatung, um „Konfektionsaware von der Stange“ und „kein maßgeschneidertes Produkt“ handelt.

80 Vgl. https://myflyright.com/de/agb/.

81 Vgl. Fabian Widder, 'Verbraucherschutz und RDG - wo bleibt die Anwaltschaft? - Legal Tech - Notwendigkeit einer gesetzlichen Regelung nach dem BGH-Urteil Lexfox' [2020] AnwBl 269.

82 So berichtet Flightright davon schon 300.000.000 Euro durchgesetzt zu haben, vgl. Homepage - https://www.flightright. $\mathrm{de} /$.

83 Vgl. Reinhard Greger, 'Streiten - oder streiten lassen? Erfolg des „Rundum-sorglos-Modells - Innovative Formen und rechtliche Grenzen der Konfliktdelegation' [2017] MDR 932; Markus Hartung, 'Legal Tech und das RDG - Raus aus der Beziehungskrise! - Warum es bei der Legal Tech-Diskussion nicht um Tech, sondern um den Zugang zum Recht geht' [2020] AnwBl 8 [9].

84 Martin Henssler, 'Prozessfinanzierende Inkassodienstleister - Befreit von den Schranken des anwaltlichen Berufsrechts?' [2019] NJW 545. Es wird primär eine Prozessfinanzierung gegen Erfolgsbeteiligung angeboten.

85 Gegen dieses Geschäftsmodell statt vieler, vgl. Martin Henssler, 'Prozessfinanzierende Inkassodienstleister - Befreit von den Schranken des anwaltlichen Berufsrechts?' [2019] NJW 545ff.

86 Siehe dazu, dass lediglich Konfektionsware angeboten werden kann, Markus Hartung, 'Legal Tech und das RDG - Raus aus der Beziehungskrise! - Warum es bei der Legal Tech-Diskussion nicht um Tech, sondern um den Zugang zum Recht geht' [2020] AnwBl 8 [8]; Wozu algorithmisierte Rechtsfindung bereits im Stande ist vgl., Martin Engel, 'Algorithmisierte Rechtsfindung als juristische Arbeitshilfe'[2014] JZ 1096. - DOI: https://doi.org/10.1628/002268814x14128399965895; Markus Hartung, Micha-Manuel Bues, Gernot Halbleib, Legal Tech - Die Digitalisierung des Rechtsmarkts (C.H. Beck 2017) 259ff.

87 In der Regel 30-35\%; Reinhard Greger, 'Streiten - oder streiten lassen? Erfolg des „Rundum-sorglos-Modells - Innovative Formen und rechtliche Grenzen der Konfliktdelegation' [2017] MDR 932; Martin Henssler, 'Prozessfinanzierende Inkassodienstleister - Befreit von den Schranken des anwaltlichen Berufsrechts?' [2019] NJW 545.

88 Vgl. Begriffsbestimmung Reinhard Greger, 'Streiten - oder streiten lassen? Erfolg des „Rundum-sorglos-Modells - Innovative Formen und rechtliche Grenzen der Konfliktdelegation' [2017] MDR 932 [933]; ders., 'Rundum-sorglos-Modell“: Innovative Rechtsdienstleistung oder Ausverkauf des Rechts?' [2018] MDR 897. 
die verhindern, dass der Einzelne Zugang zu seinen Rechten erhält, die Kostendimension ist, kann es vom Ergebnis herkommend keinen Unterschied machen, wenn marktbasierte oder staatliche Finanzierungsmechanismen diesen Zugang zum Recht gewährleisten. Begünstigt wird dieser Wandlungsprozess hin zu stärker marktbasierten Finanzierungsmechanismen auch dadurch, dass das System der Finanzierung der Kosten einer Rechtsverfolgung in sich flexibel ist. ${ }^{*}{ }^{*}$ Exemplarisch sei hier der schwedische Ansatz genannt, der die Verantwortung für die Finanzierung des Zugangs zum Recht der Versicherungswirtschaft überantwortet hat. Die staatliche Kostenhilfe konnte drastisch reduziert werden, indem die Bevölkerung auf die Möglichkeit zur Inanspruchnahme ihrer Rechtsschutzversicherung verwiesen wird. Eine solche ist in die Hausratsversicherung, über die so gut wie alle Schweden verfügen, integriert. ${ }^{*} 90$ Wie bereits angesprochen, muss die Reduzierung staatlich organisierter Finanzierungsmodelle nicht als politisch motivierte Zurücksetzung der Bedeutung der Gewährleistung des Zugangs zum Recht verstanden werden. An die Stelle des Staates tritt vielmehr als Finanzierer der Rechtsverfolgung die freie Marktwirtschaft und es findet sozusagen die Privatisierung einer Aufgabe statt, die traditionell als staatlich verstanden wird. ${ }^{*} 91$ Problematisch ist eine solche Aufgabenüberantwortung dann, wenn dadurch verfassungsrechtlich verbürgte Gemeinschaftsgüter gefährdet werden, die dem Schutz eines modernen Sozial- und Rechtsstaates unterstehen. ${ }^{*} 2$

\section{Nationale und internationale marktimmanente Barrieren, die der Hinwendung zu marktbasierten Finanzierungsmechanismen entgegenstehen}

Der Rechtsdienstleistungsmarkt in Estland ist vollends dereguliert. Bis auf die Prozessvertretung vor dem Obersten Gerichtshof sowie bei der Übernahme eines Mandats, die mit staatlichen Mitteln finanziert werden, stehen estnische Rechtsanwälte im freien Wettbewerb mit nichtanwaltlichen Rechtsdienstleistern. ${ }^{*} 93$ Demgegenüber ist der deutsche Rechtsdienstleistungsmarkt seit dem 13. Dezember $1935^{*}{ }^{94}$ durch das damals verkündete Rechtsberatungsmissbrauchsgesetz (RBerMißG) protektioniert. ${ }^{*} 95$ Nachdem das RBerMißG unter der Überschrift Rechtsberatungsgesetz (RBerG) in die amtliche Sammlung des Bundesrechts inhaltlich weitgehend ${ }^{*} 96$ unverändert aufgenommen wurde ${ }^{*} 97$, finden sich heute Befugnisse für die Teilnahme am deutschen Rechtsdienstleistungsmarkt im nach der Reform des RBerG am 01. Juli 2008 ${ }^{*} 98$ in Kraft getretenen Rechtsdienstleistungsgesetz (RDG). Nach wie vor steht somit eine unter dem RBerG genannte Rechtsbesorgung, heute Rechtsdienstleistung, unter einem präventiven Verbot mit Erlaubnisvorbehalt, dass $\S 3$ in Verbindung mit $\S 2$ Abs. 1 und 2 RDG statuiert; nur derjenige, der über eine Erlaubnis verfügt oder von der Erlaubnispflicht frei ist, darf damit Tätigkeiten im Sinne von außergerichtlichen (Rechts-) Dienstleistungen erbringen und am Rechtsdienstleistungsmarkt anbieten. ${ }^{*} 99$ Auch wenn dies

89 Matthias Kilian, 'Zugang zum Recht: Beobachtungen in Zeiten von Gesetzen zur Kosteneinsparung bei der Prozesskostenund Beratungshilfe' [2008] AnwBl 236 [239].

90 Ibid 239f.

91 Ähnlich, jedoch in Bezug auf ein anwaltliches Erfolgshonorar, Matthias Kilian, 'Zugang zum Recht: Beobachtungen in Zeiten von Gesetzen zur Kosteneinsparung bei der Prozesskosten- und Beratungshilfe' [2008] AnwBl 236 [239].

92 Insbesondere wenn der Verbraucherschutz dadurch leidet, vgl. dazu Schriftliche Stellungnahme zum Entwurf eines Gesetzes zur Förderung verbrauchergerechter Angebote im Rechtsdienstleistungsmarkt Prof. Dr. Christian Wolf, Ass. Iur. Nadja Flegler - https://www.bmjv.de/SharedDocs/Gesetzgebungsverfahren/Stellungnahmen/2020/Downloads/122120_Stellungnahme_IPA_RefE_Rechtsdienstleistungsmarkt.pdf;jsessionid=97FD7B32FEB5155A0AD2FB9F6301D287.1_cid324?_ blob=publicationFile\&v=2, S. 11f; zuletzt abgerufen am 14.01.2021.

93 Vgl. Deutscher Bundestag Drucks. 16/3655, S. 30 - http://dip21.bundestag.de/dip21/btd/16/036/1603655.pdf; zuletzt abgerufen am 14.01.2021.

94 RGBl.IS. 1478 - https://upload.wikimedia.org/wikipedia/commons/1/18/Deutsches_Reichsgesetzblatt_35T1_140_1478. jpg; zuletzt abgerufen am 14.01.2021.

95 Zuvor, unterlag die gewerbsmäßige Besorgung fremder Rechtsangelegenheiten den Bestimmungen der GewO, vgl. Günter Rennen, Gabriele Caliebe in dies. (Hrsg.), Rechtsberatungsgesetz (C.H. Beck 1986) Art. $1 \S 1$ Rn. 1.

96 Eine Änderung vor der Namensänderung erfuhr, das ursprüngliche Rechtsberatungsmissbrauchsgesetz v. 13.12.1935 (RGBl. I S. 1478) durch das Außenwirtschaftsgesetz v. 28.04.1961 (BGBl. I S. 481 (493)).

97 BGBl. III 303-12.

98 Vgl. Art. 20 Gesetz zur Neuregelung des Rechtsberatungsrechts v. 12.12.2007 BGBl. I S. 2860.

99 Vgl. Frank Remmertz in Michael Krenzler (Hrsg.), Rechtsdienstleistungsgesetz (2. Auflage, Nomos 2017) § 1 Rn. 6. 
immer wieder behauptet wird ${ }^{* 100}$, stellt dieser rechtserhebliche Eingriff in die durch Art. 12 Abs. 1 des Grundgesetz geschützte Ebene der objektiven Berufswahlfreiheit ${ }^{*}{ }^{* 101}$ oder zumindest der Berufsausübungsfreiheit ${ }^{*}{ }^{* 12}$ keine Ausnahme im europäischen Vergleich, dar. ${ }^{*}{ }^{* 103}$ Außer in Estland und Schweden ${ }^{* 104}$ finden sich in jedem anderen Mitgliedstaat der EU mal mehr ${ }^{*}{ }^{* 105}$, mal weniger vergleichbare Ausformungen eines Rechtsdienstleistungsmonopols. ${ }^{* 106}$ In diesem Kontext ist auch die Entwicklung in Frankreich hervorzuheben, wo durch Art. 54 neue Fassung Rechtsanwaltsgesetz (RAG) zum 01.01.1992 erstmals ein Rechtsberatungsmonopol eingeführt wurde. ${ }^{*} 107$ Als überragend wichtige Gemeinschaftsgüter, die einen Eingriff in die Berufswahlfreiheit rechtfertigen, sind heute noch ${ }^{* 108}$ die in § 1 Abs. 1 RDG explizit aufgeführten Ziele, die Rechtssuchenden, den Rechtsverkehr und damit die Rechtsordnung vor unqualifizierten Rechtsdienstleistungen zu schützen, anzusehen. Mag das Rechtsdienstleistungsgesetz damit zwar in seiner Funktion und Zielsetzung ehrbaren Zwecken dienen, wird seit nunmehr fast vierzig Jahren ${ }^{*} 109$ in der deutschen Literatur vehement über dessen Daseinsberechtigung und seine Reichweite gestritten. ${ }^{*} 110$ Seit zwanzig Jahren spielt dabei auch die dem RDG immanente Behinderung des Zugangs zum Recht ${ }^{*}{ }^{111}$ eine immer größer werdende Rolle. Denn wenn sich bei der Verbesserung des Zugangs zum Recht vermehrt den marktbasierten Finanzierungsmechanismen zugewandt werden soll, stellen derartige Beschränkungen eine zusätzliche Barriere auf dem Weg dorthin dar. Auch dieser Aspekt muss bei der Frage berücksichtigt werden, ob das Gesetz noch als „erforderlich“ angesehen werden kann, um die verfassungsrechtlich als gemeinwohlrelevant qualifizierten Ziele des Rechtsdienstleistungsgesetzes in der Form des Schutzes des Rechtssuchenden und der ordnungsgemäßen Rechtspflege nachhaltig schützen zu können. ${ }^{*}{ }^{112}$

Da unabhängig davon, ob die Dienstleistungen der oben beschriebenen Geschäftsmodelle unter die Legaldefinition des Rechtsdienstleistungsbegriffs des § 2 Abs. 1 RDG fallen, der bestimmt, dass eine Rechtsdienstleistung ,jede Tätigkeit in konkreten fremden Angelegenheiten ist, sobald sie eine rechtliche Prüfung des Einzelfalls erfordert“, unterfallen Inkassodienstleistungen im Sinne des § 2 Abs. 2 RDG, also die Einziehung fremder oder zum Zweck der Einziehung auf fremde Rechnung abgetretene Forderungen, dem präventiven Verbot mit Erlaubnisvorbehalt des § 3 in Verbindung mit § 2 Abs. 2 RDG. ${ }^{*}{ }^{113}$ Für diese Art der Rechtsdienstleistung ist damit der materielle Anwendungsbereich des RDG eröffnet und es ist in einem nächsten Schritt zu ermitteln, ob derjenige, der eine derartige außergerichtliche (Rechts-)Dienstleistung erbringt und am Rechtsdienstleistungsmarkt anbieten möchte, über eine Erlaubnis verfügt oder von der Erlaubnispflicht frei ist. Gemäß § 3 Bundesrechtsanwaltsordnung (BRAO) sind die Anwälte, als berufene

100 Michael Kleine-Cosack, 'Vom Rechtsberatungsmonopol zum freien Wettbewerb - Erosion des Rechtsberatungsgesetzes' [2000] NJW 1593 [1594, 1597].

101 Ein derartiger Eingriff kann nur durch die Abwehr nachweisbarer oder höchstwahrscheinlich schwererer Gefahren für ein überragend wichtiges Gemeinschaftsgut gerechtfertigt werden, vgl. BVerfGE 97,12; NJW 1998, 3481 [3481].

102 Ein Eingriff in die Berufsausübungsfreiheit, kann hingegen schon aufgrund eines Interesses des Gemeinwohls gerechtfertigt sein.

103 Vgl. Überblick bei Bernhard Dombek, “'Rechtsberatungsmonopol” der Anwaltschaft - Berechtigung und Grenzen’ [2001] BRAK-Mitt. 98 [100].

104 Deutscher Bundestag Drucks. 16/3655, S. 28.

105 Vgl. für Österreich, das damit die gleichen Regeln wie Deutschland hat, Bernhard Dombek, “Rechtsberatungsmonopol” der Anwaltschaft - Berechtigung und Grenzen' [2001] BRAK-Mitt. 98 [101].

106 Michael Kleine-Cosack in ders. (Hrsg.), Rechtsdienstleistungsgesetz (C.F. Müller 2014), Allg. Teil Rn. 19.

107 Vgl. Bernhard Dombek, “'Rechtsberatungsmonopol” der Anwaltschaft - Berechtigung und Grenzen' [2001] BRAK-Mitt. 98 [101].

108 Bis zum Masterpatent-Beschluss des BVerfG NJW 1998, 3481 (3483) war originäres 3 Ziel des RBerG, der Anwaltschaft ein ausreichendes Arbeitsfeld zu sichern als Gegenleistung für Ausbildung und berufsständische Bindungen, vgl. Begründung zum Rechtsberatungsgesetz abgedruckt in, Rudolf Altenhoff, Hans Busch, Kurt Kampmann in dies. (Hrsg.), Rechtsberatungsgesetz (Aschendorff, Münster Westfalen 1973) Anhang B S. 238ff.

109 Insbesondere nach dem die unter der alten Rechtslage bestehende Möglichkeit, eine unbeschränkte Vollerlaubnis als Rechtsbeistand zu beantragen, durch das 5. BRAGebÄndG vom 18.08.1980 aufgehoben wurde - was zu einer Verschärfung der Monopolstellung führte - da seitdem nur noch eine Teilerlaubnis in abschließend aufgezählten Rechtsgebieten erteilt werden konnte, vgl. Günter Rennen, Gabriele Caliebe in dies. (Hrsg.), Rechtsberatungsgesetz (C.H. Beck 1986) Art. 1 § 1 Rn. 3.

110 Vgl. statt vieler, Volker Römermann, 'RDG - zwei Schritte vor, einen zurück' [2008] NJW 1249; a.A. Kleine-Cosack, 'Öffnung des Rechtsberatungsmarkts - Rechtsdienstleistungsgesetz verabschiedet' [2007] BB 2637.

111 Martin Henssler, ‘Die Zukunft des Rechtsberatungsgesetzes' [2001] AnwBl. 525 [526].

112 Vgl. hierzu noch zum alten RBerG Michael Kleine-Cosack, 'Vom Rechtsberatungsmonopol zum freien Wettbewerb - Erosion des Rechtsberatungsgesetzes' [2000] NJW 1593 [1596].

113 BVerfG NJW 2020, 208 [213] Rn. 40ff. 
Berater und Vertreter in allen Rechtsangelegenheiten, folgelogisch von der Erlaubnis- d.h. Registrierungspflicht des RDG befreit. ${ }^{*}{ }^{114}$ Für nicht-anwaltliche Anbieter von Inkassodienstleistungen besteht daneben im Rahmen des RDG die Möglichkeit, eine Erlaubnis, in der Form der Registrierung bei der zuständigen Behörde gemäß § 10 Abs. 1 Nr. 1 RDG, als Inkassodienstleister zu erhalten. ${ }^{*} 115$ Hierfür müssen neben anderen Voraussetzungen, die unter anderem die Verordnung zum Rechtsdienstleistungsgesetz (RDV) statuiert, insbesondere ein Sachkundenachweis gem. § 12 RDG in Verbindung mit § 4 RDV erbracht werden ${ }^{* 116}$ sowie die Eintragung in das Rechtsdienstleistungsregister gemäß § 16 RDG erfolgen.

Da die Geschäftsmodelle unstreitig auch eine Inkassodienstleistung erbringen, der sonstige Dienstleistungsumfang der Unternehmen sich jedoch vom klassischen Inkassobild - massenhafte Geltendmachung von grundsätzlich unstreitigen Forderungen durch Unternehmen gegen säumige Verbraucher ${ }^{* 117}$ - deutlich unterscheidet, wurde und wird in der Folge verstärkter Verbreitung derartiger Unternehmen in der deutschen Literatur und Rechtsprechung ${ }^{* 118}$ heftig über deren Zulässigkeit diskutiert. ${ }^{* 19}$ Neben anderen $^{*}{ }^{120}$ insbesondere unter dem Gesichtspunkt, ob das gesamte Geschäftsmodell der Unternehmen in diesem Umfang von der grundsätzlich erteilten Erlaubnis gemäß § 10 Abs. 1 Nr. 1 RDG (noch) umfasst sein kann. ${ }^{*}{ }^{121}$ Angeheizt wurde diese Debatte vor allem durch die, nach herrschender Literaturansicht ${ }^{{ }^{*} 122}$, allein den Inkassodienstleitern zustehende Möglichkeit, eine erfolgsabhängige Vergütung für die Rechtssuchenden anbieten zu können. ${ }^{*} 123$ Gemäß $\S 49$ b Abs. II S. 1 BRAO in Verbindung mit § 4 a RVG ist dies den Rechtsanwälten grundsätzlich verwehrt. ${ }^{{ }^{1} 124}$

Auf die Einzelheiten dieser umfassenden Debatte kann im Rahmen dieses Beitrages nicht näher eingegangen werden. ${ }^{*}{ }^{125}$ Festgehalten werden sollte aber, dass der Bundesgerichtshof (BGH) in einer Leitentscheidung zur Vereinbarkeit des Dienstleistungsumfangs, der Plattform von „wenigermiete.de“ der Conny- vormals LexFox-GmbH, mit dem RDG im Jahr 2019 zu der Ansicht gelangte, dass das explizite Geschäftsmodell der Conny-GmbH, zwar dem Erlaubnisvorbehalt des Rechtsdienstleistungsgesetzes unterfällt, jedoch im Rahmen der Registrierung als Inkassodienstleister gemäß § 10 I 1 Nr. 1 RDG sich (noch) als erlaubnisfähiger Teilnehmer am Rechtsdienstleistungsmarkt erweise und damit beteiligen dürfe. ${ }^{*} 126$ Zwar wurde damit keine allgemeingültige Aussage über die Zulässigkeit anderer Geschäftsmodelle getroffen. ${ }^{*}{ }^{127}$ Dennoch verwies der Bundesgerichtshof in seinem Urteil mit Nachdruck darauf, dass der RBerG-Reformgesetzgeber von 2008 erhebliche Deregulierungs- und Liberalisierungsbestrebungen ${ }^{* 128}$ des

114 Frank Remmertz in Michael Krenzler (Hrsg.), Rechtsdienstleistungsgesetz (2. Auflage, Nomos 2017) § 1 Rn. 7.

115 Vgl. Daniela Schmidt in Michael Krenzler (Hrsg.), Rechtsdienstleistungsgesetz (2. Auflage, Nomos 2017) § 10 Rn. 6.

116 Hierfür ist gemäß $\S 4$ Abs. 1 RDV ein Lehrgang von 120 Zeitstunden sowie nach § 4 Abs. 3 RDV der Nachweis mindestens einer bestandenen fünfstündigen Klausur notwendig.

117 Markus Hartung, 'Legal Tech und das RDG - Raus aus der Beziehungskrise! - Warum es bei der Legal Tech-Diskussion nicht um Tech, sondern um den Zugang zum Recht geht' [2020] AnwBl 8 [9].

118 Zur Unzulässigkeit des Modells, Landgericht (LG) Berlin BeckRS 2018, 19885; LG München AnwBl 2020, 238 [239]; LG Hannover, NZKart, 2020, 398, 400; zur Zulässigkeit, LG Berlin NJW 2018, 2898; LG Berlin BeckRS 2018, 18018.

119 Befürworter statt vieler, Markus, Hartung, 'Inkasso, Prozessfinanzierung und das RDG - Was darf ein Legal-Tech-Unternehmen als Inkassodienstleister?” [2019] AnwBl 353; Gegner statt vieler, Reinhard Greger, 'Rundum-sorglos-Modell“: Innovative Rechtsdienstleistung oder Ausverkauf des Rechts?' [2018] MDR 897.

120 Zu "MyRight" und der Problematik des Interessenskonflikt gemäß § 4 RDG vgl., Martin Henssler, 'Prozessfinanzierende Inkassodienstleister - Befreit von den Schranken des anwaltlichen Berufsrechts?' [2019] NJW 545; andere Ansicht Alexander Morell, 'Keine Kooperation ohne Konflikt' [2019] JZ 809 [814]. - DOI: https://doi.org/10.1628/jz-2019-0299.

121 Instruktiv Fabian Widder, 'Verbraucherschutz und RDG - wo bleibt die Anwaltschaft? - Legal Tech - Notwendigkeit einer gesetzlichen Regelung nach dem BGH-Urteil Lexfox' [2020] 269.

122 Günther Bandisch in Walter Seitz (Hrsg.), Inkasso-Handbuch (4. Auflage, C.H. Beck 2015) Kapitel 31 Rn. 111; Dirk Seichter in Christian Deckenbrock und Martin Henssler (Hrsg.), RDG (4. Auflage, C.H. Beck 2015) § 4 RDGEG Rn. 4.

123 A.A. Martin Henssler, 'Prozessfinanzierende Inkassodienstleister - Befreit von den Schranken des anwaltlichen Berufsrechts?' [2019] NJW 545 [548]; Klaus Winkler in Michael Krenzler (Hrsg.), Rechtsdienstleistungsgesetz (2. Auflage, Nomos 2017) $\S 4$ RDGEG Rn. 11.

124 Matthias Kilian in Martin Henssler und Hanns Prütting (Hrsg.), BRAO (5. Auflage, C.H. Beck 2019) § 49b BRAO Rn. 72ff, $101 \mathrm{ff}$.

125 Überblick, wer Gutachtertätigkeiten für die entgegengesetzten Lager übernommen hat, Alexander Morell, 'Keine Kooperation ohne Konflikt' [2019] JZ 809 [809 Fn 4].

126 BGH NJW 2020, 208 (213) Rn. 38.

127 So betont der BGH NJW 2020, 208 [221] Rn. 110, dass es stets einer Würdigung der Umstände des Einzelfalls bedürfe; ebenso Robert Freitag, David Lang, 'Offene Fragen von Legal und Illegal Tech nach der wenigermiete.de-Entscheidung" des BGH' [2020] ZIP 1201 [1210].

128 Kritisch dazu Volker Römermann, 'RDG - zwei Schritte vor, einen zurück' [2008] NJW 1249. 
Rechtsdienstleistungsmarktes im Blick hatte, die die Entwicklung neuer Berufsbilder erlauben und denen es Rechnung zu tragen gilt. ${ }^{*}{ }^{* 129}$ Es bleibt abzuwarten, ob der deutsche Gesetzgeber diese Rechtsprechung in neuen Reformbestrebungen bestätigen und weiter ausbauen und damit den Rechtsdienstleistungsmarkt für weitere Finanzierungsmechanismen öffnen wird. ${ }^{{ }^{*} 30}$

Der aktuelle deutsche rechtspolitische Diskurs geht derweil noch einen Schritt weiter. So wird diskutiert ${ }^{* 131}$, ob der Anwaltschaft im größeren Umfang als bisher die Vereinbarung von Erfolgshonoraren ermöglicht werden sollen. Zwar sind in den letzten 20 Jahren in vielen europäischen Rechtsordnungen Erfolgshonorare legalisiert worden und unstreitig ließe sich damit die staatliche Kostenhilfe noch einmal deutlich reduzieren. ${ }^{* 132}$ Als unabhängiges Organ der Rechtspflege begegnet dieser Ansatz aber erheblichen Bedenken in der Literatur und daneben auch aus den Reihen der Anwaltschaft selbst.

\section{Resümee}

Die von Mauro Cappelletti formulierte Permisse von 1978, dass man sich im langen historischen Kampf um den Zugang zum Recht befinde, hat an ihrer Aktualität nicht eingebüßt. Vielmehr scheint es, dass sich der Kampf, um den - finanzierbaren - Zugang zum Recht gegenwärtig noch verstärkt hat. Nach der Abkehr vom Ausbau staatlich organisierten Finanzierungsmodellen etablieren sich vermehrt marktbasierte Finanzierungsmechanismen außerhalb der Anwaltschaft, deren Mitglieder traditionell als berufene Berater und Vertreter in allen Rechtsangelegenheiten angesehen wurden. Durch genaue Bedürfnisanalysen der rechtssuchenden Bevölkerung verstehen es die neuen Rechtsdienstleister gut, sich weiteres Marktpotenzial zu erschließen. Dies gelingt ihnen auch dadurch besser, da sie sich, sobald sie als Inkassodienstleister gemäß $\S 10$ Nr. 1 RDG registriert sind, zur Bedürfnisbefriedigung Mitteln bedienen dürfen, die der Anwaltschaft bis dato - vielleicht auch berechtigter Weise ${ }^{*}{ }^{133}$ - verwehrt bleiben. Es bleibt abzuwarten, ob die Rechtsprechung und der Gesetzgeber diesen Deregulierungs- und Liberalisierungsansätzen - weiter - folgen werden. Oder aber, ob in Zukunft, der bestehende Wettbewerbsvorteil ausgleichen wird und als Finanzierer der Rechtsberatung und der Rechtsdurchsetzung vermehrt auch den Rechtsanwalt treten darf. ${ }^{*}{ }^{134}$ Bei der Delegation einer derartigen verfassungsrechtlich verbürgten staatlichen Aufgabe ${ }^{* 135}$ sollte in einem Sozialstaat aber immer auch ein besonderes Augenmerk auf den Umstand gelegt werden, dass tatsächlich alle Rechtssuchenden den - marktbasierten - Zugang zum Recht erhalten. Denn dieser folgt anderen, nach Eigenkapital kalkulierten Grundsätzen ${ }^{* 136}$ und nimmt im Zweifel auf atypische Sachverhalte sowie bildungsschwächere Bevölkerungsgruppen, die einer besonderen Hilfestellung beim Auffinden und Erkennen eines rechtlichen Konflikts bedürfen, keine Rücksicht. Auch in den nächsten vierzig Jahren werden noch einige Kämpfe geführt werden müssen auf dem Weg hin zu einem Rechtssystem, das für jedermann gleichermaßen zugänglich ist.

129 BGH NJW 2020, 208 (223) Rn. 133.

130 Appell, dass der Gesetzgeber tätig werden, muss auch von Robert Freitag, David Lang, 'Offene Fragen von Legal und Illegal Tech nach der wenigermiete.de-Entscheidung“ des BGH’ [2020] ZIP 1201 [1210].

131 Vgl. Referentenentwurf des Bundesministerium der Justiz und für Verbraucherschutz Entwurf eines Gesetz zur Förderung verbrauchergerechter Angebote im Rechtsdienstleistungsmarkt vom 12.11.2020 - https://www.bmjv.de/SharedDocs/ Gesetzgebungsverfahren/Dokumente/RefE_Rechtsdienstleister.pdf?_blob=publicationFile\&v=1; zuletzt abgerufen am 14.01.2021.

132 Vgl. Matthias Kilian, 'Zugang zum Recht: Beobachtungen in Zeiten von Gesetzen zur Kosteneinsparung bei der Prozesskosten- und Beratungshilfe' [2008] AnwBl 236 [239 mwN].

133 Gem. § 1 BRAO ist der Rechtsanwalt ein unabhängiges Organ der Rechtspflege.

134 Siehe dazu Referentenentwurf des Bundesministeriums der Justiz und für Verbraucherschutz Entwurf eines Gesetzes zur Förderung verbrauchergerechter Angebote - https://www.bmjv.de/SharedDocs/Gesetzgebungsverfahren/Dokumente/ RefE_Rechtsdienstleister.pdf?_blob=publicationFile\&v=1; zuletzt abgerufen am 14.01.2021.

135 Der Justizgewährungsanspruch folgt aus dem Rechtsstaatsprinzip Art. 20 III GG und wird ebenso aus dem Fair-trail-Grundsatz abgeleitet Art. 6 I EMRK; dazu BVerfGE 107, 395, vgl. oben Fn 67, 68 und 23, 24.

136 Schriftliche Stellungnahme zum Entwurf eines Gesetzes zur Förderung verbrauchergerechter Angebote im Rechtsdienstleistungsmarkt Prof. Dr. Christian Wolf, Ass. Iur. Nadja Flegler - https://www.bmjv.de/SharedDocs/Gesetzgebungsverfahren/ Stellungnahmen/2020/Downloads/122120_Stellungnahme_IPA_RefE_Rechtsdienstleistungsmarkt.pdf;jsessionid=97FD 7B32FEB5155A0AD2FB9F6301D287.1_cid324?__blob=publicationFile\&v=2 S. 5, 11ff; zuletzt abgerufen am 14.01.2021. 